\title{
MOVING: Motivation-Oriented interVention study for the elderly IN Greifswald: study protocol for a randomized controlled trial
}

\author{
Fabian Kleinke ${ }^{1,2^{*}}$, Thea Schwaneberg ${ }^{1,2}$, Franziska Weymar ${ }^{1,2,3}$, Peter Penndorf ${ }^{1,2}$, Sabina Ulbricht ${ }^{2,3}$, \\ Kristin Lehnert ${ }^{2,4}$, Marcus Dörr ${ }^{2,4}$, Wolfgang Hoffmann ${ }^{1,2}$ and Neeltje van den Berg ${ }^{1,2}$
}

\begin{abstract}
Background: Cardiovascular diseases (CVD) are the leading cause of mortality. In 2014, they were responsible for 38 . $9 \%$ of all causes of death in Germany. One major risk factor for CVD is a lack of physical activity (PA). A healthpromoting lifestyle including regular PA and minimizing sitting time (ST) in daily life is a central preventive measure. Previous studies have shown that PA decreases in older age; 2.4-29\% of the people aged over 60 years achieve the World Health Organization recommendations. This age group spends on average $9.4 \mathrm{~h}$ per day in sedentary activities. To increase PA and decrease ST, a low-threshold intervention, consisting of individualized feedback letters based on objectively measured data of PA and ST, was developed. The research question is: Do individual feedback letters, based on accelerometer data, have a positive effect on PA and ST?
\end{abstract}

Methods/design: MOVING is a two-arm, randomized controlled trial. Inclusion criteria are age $\geq 65$ years and the ability to be physically active. Exclusion criteria are the permanent use of a wheelchair and simultaneous participation in another study on PA. At baseline participants who give informed consent will receive general information and recommendations about the positive effects of regular PA and less ST. Participants of both groups will receive an accelerometer device, which records PA and ST over a period of seven consecutive days following by a randomization. Participants in the intervention group will receive automatically generated, individualized feedback letters by mail based on their PA and ST at baseline and at 3-month follow-up. Further follow-up examinations will be carried out at 6 and 12 months. The primary outcome is the increase of PA and the reduction of ST after 6 months in the intervention group compared to the control group.

Discussion: The goal of the study is to examine the effects of a simple feedback intervention on PA and ST in elderly people. We aim to achieve an effect of $20 \%$ increase in moderate-to-vigorous physical activity (MVPA). The intervention may have the potential to decrease crucial cardiovascular risk factors and, therefore, contribute to prevention of CVD.

Trial registration: German Clinical Trials Register, ID: DRKS00010410. Registered on 17 May 2017.

Keywords: Physical activity, Sedentary behavior, Accelerometry, Elderly individuals (people), motivation, Behavior change, Intervention, RCT

\footnotetext{
* Correspondence: fabian.kleinke@uni-greifswald.de

${ }^{1}$ Institute for Community Medicine, Section Epidemiology of Health Care and

Community Health, University Medicine Greifswald, Greifswald, Germany

2DZHK (German Centre for Cardiovascular Research), partner site Greifswald,

Greifswald, Germany

Full list of author information is available at the end of the article
}

(c) The Author(s). 2018 Open Access This article is distributed under the terms of the Creative Commons Attribution 4.0 International License (http://creativecommons.org/licenses/by/4.0/, which permits unrestricted use, distribution, and reproduction in any medium, provided you give appropriate credit to the original author(s) and the source, provide a link to the Creative Commons license, and indicate if changes were made. The Creative Commons Public Domain Dedication waiver (http://creativecommons.org/publicdomain/zero/1.0/) applies to the data made available in this article, unless otherwise stated. 


\section{Background}

Older age is associated with increasing risk for chronic diseases and multimorbidity [1-3]. The prevalence of cardiovascular diseases (CVD), including coronary heart disease (CHD) and stroke, increases with age [4]. In 2014, CVD were the leading cause of mortality [5] and responsible for $38.9 \%$ of all causes of death in Germany [6]. CVD produce substantial disabilities and reduce well-being $[7,8]$. Therefore, older people are a relevant target group for effective and practicable strategies for CVD prevention [9].

According to the World Health Organization (WHO), lack of PA is an important risk factor for mortality [10] and is associated with a series of non-communicable diseases (NCD), e.g., type 2 diabetes and a number of cancers (e.g., colon cancer and breast cancer) [7]. Several studies have shown that a health-promoting lifestyle, including promotion of PA and reduction of ST, is correlated with improved overall health status [7, 11]. Furthermore, regular PA is a resource for physical and mental health and reduces risks of obesity and musculoskeletal system problems. Regular PA significantly decreases overall mortality by $22-34 \%$ and CVD mortality by $27-35 \%$ [12].

In general, international PA guidelines do not recommend that elderly people do less PA than younger adults [13-15]. The WHO recommends $150 \mathrm{~min}$ of moderate or $75 \mathrm{~min}$ of vigorous $\mathrm{PA}$, or a corresponding combination of weekly PA for both for younger adults (aged 18-64 years) and older people aged over 65 years. PA should be done in time periods (bouts) of at least $10 \min [14]$.

Recent academic literature points out that PA decreases in older age [16-18] while the amount of ST increases to high levels (about an average of $60 \%$ ST of waking time) $[19,20]$. In Germany, $19.3 \%$ of men and $16.8 \%$ of women aged $60-69$ years achieved the WHO recommendations. In the age group 70-79 years the amount of PA declines to $16.5 \%$ of men and $11.0 \%$ of women [10]. A study from the United Kingdom showed that $19 \%$ of men and $14 \%$ of women aged $65-74$ years reach the recommended $30 \mathrm{~min}$ of moderate PA at least five times a week [7]. Data from Norway showed similar results among older people: $29 \%$ of men and $25 \%$ of women aged 65-69 years met the Norwegian PA recommendations (minimum of $30 \mathrm{~min}$ of daily PA of moderate intensity). In the age group $80-85$ years the amount of PA declines; $7.1 \%$ of women and $3 \%$ of men reached the recommendations [9].

The National Health and Nutrition Survey (NHANES, USA) showed that $2.4 \%$ of people aged over 60 years achieved WHO recommendations for PA [21]. This age group spends on average $8.4 \mathrm{~h}(60-69)$ and $9.3 \mathrm{~h}$ (70-85) per day in sedentary activities (measured by accelerometer) which correspondents up to $70 \%$ of their wake time [19]. A systematic review of adults aged 60 years and older showed similar results: older people spend an average of $9.4 \mathrm{~h}$ doing sedentary activities which corresponds to $65-80 \%$ of their waking day. It is particularly striking that estimated self-reported ST is lower than objectively measured: on average $5.3 \mathrm{~h}$ per day [22]. In the aforementioned Norwegian study participants aged 65-85 years spend $66 \%$ of the day in ST, $24 \%$ doing low-intensity PA and 3\% doing moderate-to-vigorous physical activity (MVPA) [9].

The variation in the data can be explained by the fact that studies are conducted in different settings and the definition of elderly people varies. Furthermore, there are differences in the assessment of PA data. Some studies are based on measured accelerometer data, others on self-reported data. PA assessment based on selfreporting methods may not quantify PA levels correctly [7]. The use of accelerometer devices for objective assessment of PA allows a valid and reliable record of activity intensity, frequency, and duration which may improve our understanding of PA in the elderly [7, 23].

Individualized feedback on accelerometer measurements can help to increase PA and reduce ST [24-26]. Interventions in promoting PA should consider behavioral-change aspects. A systematic review regarding interventions to promote walking identified two general characteristics of effective interventions. These aspects are targeting and tailoring and involve addressing participants' requirements or circumstances [27].

Harris et al. showed statistically significant positive effects in the age group 60-75 years on the amount of PA (average daily step-counts and weekly MVPA in $\geq 10$-min bouts) [26]. This intervention included four primary care nurse consultations during a period of 3 months. Participants received feedback on the basis of pedometer step-counts, accelerometer PA intensity, and an individual PA diary and plan. Furthermore, a meta-analyses regarding the impact of pedometer-based physical activity interventions showed that the use of pedometers has a moderate positive effect on PA (an average increase of 2000 steps per day [28]. A systematic review of the use of pedometer interventions showed similar results; participants increased their PA by 2491 steps per day in comparison to the control group [29].

Most previous studies have been characterized by rather complex interventions and also included feedback of PA and ST results, personal consultations, and individual target agreements. A translation of such interventions into real life may be difficult due to considerable costs and effort to reach a large number of people.

Hence, we developed a low-threshold intervention consisting of automatically retrieved, individualized feedback letters, based on accelerometer data. In a randomized 
controlled trial, the effect of this feedback intervention on the amount of PA and ST will be examined prospectively.

The study protocol follows the Standard Protocol Items: Recommendations for Interventional Trials (SPIRIT) guidelines Additional file 1 [30].

\section{Research objectives}

The primary objective of the MOVING study is to examine the effects of an increase of PA and a reduction of ST after 6 months in the intervention group compared to the control group as assessed by accelerometry.

Secondary outcomes are whether there is an improvement of blood pressure, a reduction in weight and waist circumference as well as an increase of self-reported time doing PA, self-reported ST, and self-reported time doing PA as well as the effect of feedback letters on selfefficacy after 6 and 12 months.

Additionally, the impact of personal feedback letters on lifestyle (household, gardening, free time, and sport activities), quality of life and type of PA will be evaluated by questionnaire in the intervention group compared to control group after 6 and 12 months.

\section{Methods/design}

The study region is Western Pomerania, a rural area in the Northeast of Germany.

MOVING (Motivation-Oriented interVention study for the elderly IN Greifswald) is a two-arm, randomized controlled trial consisting of participant screening, baseline examination, randomization, intervention, and follow-up examinations at 3, 6, and 12 months after baseline. The flow chart of the study is shown in Fig. 1.

\section{Study population}

The study population consists of elderly people sampled from the general population who meet the following inclusion criteria:

1. Age $\geq 65$ years

2. The possibility of being physically active in daily life

Exclusion criteria are:

1. Permanent use of a wheelchair (no ability to walk independently)

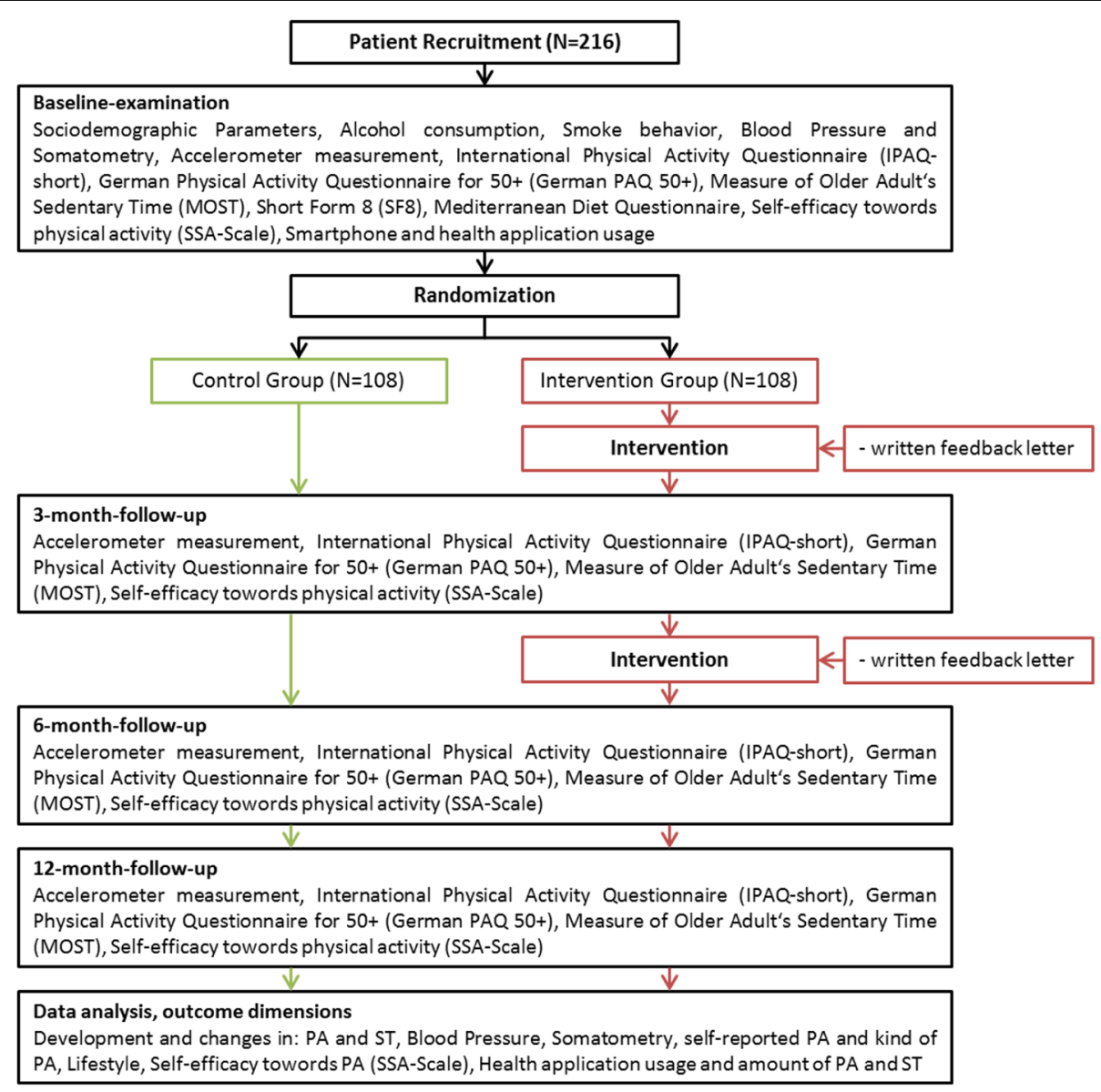

Fig. 1 Flow chart of the study 
2. Simultaneous participation in other studies including PA or ST

3. Not accessible by telephone or cell phone (necessary for screening)

4. Fulfillment of the WHO recommendations for PA (self-report) for people aged $\geq 65$ years at baseline

\section{Recruitment and screening}

Participants will be recruited in three ways:

1. Re-contacting participants of a previous study. Participants have given their consent to recontacting for scientific projects

2. Active recruitment in general medical practices (GP)

3. Recruitment of volunteers (e.g., in medical practices, hospitals, meeting centers, and via flyers and posters)

Potential participants contact the study staff by telephone or by use of a reply card with contact information. A short telephone screening of potential study participants is performed to assess the inclusion and exclusion criteria. The data will be documented in a documentation system based on electronic Case Report Forms (eCRFs). For participants who meet the inclusion criteria, an appointment for the baseline examination at the examination center of the German Centre for Cardiovascular Research (DZHK) Greifswald is made.

\section{Baseline examination}

At the examination center, the participants are informed in detail about the study and invited to give written informed consent. Upon consent all receive a baseline examination consisting of blood pressure assessment and somatometry data (body weight, waist and hip circumference). All measurements are carried out by trained and certified study staff according to standard operating procedures (SOP).

In addition, all participants receive paper-pencil questionnaires to assess sociodemographics, general health, and actual PA behavior. To asses health status and quality of life the Short Form 8 (SF-8) questionnaire (German version) is used, which consists of eight scales addressing mental and physical health [31].

Furthermore, all study participants will receive the SSA scale (self-efficacy towards physical exercise) to assess each participant's level of self-efficacy (twelve items which can be summed to a total score in which higher results indicate higher self-efficacy towards PA) [32]. To assess dietary habits, the Mediterranean Diet questionnaire (14 items, score ranging from 0 to 14) will be used [33]. Alcohol consumption will be assessed by using two items from the Alcohol Use Disorders Identification Test (AUDIT) [34].
After answering the questionnaires, all participants receive an accelerometer device (ActiGraph wGT3X-BT, Pensacola, FL, USA). The validated three-axis accelerometer device captures and records continuously PA and ST. Participants are instructed to wear the device during a period of seven consecutive days on the right hip and to remove it only for water-based activities (e.g., showering and swimming) as well as at bedtime. Data will be recorded at a sampling frequency of $30 \mathrm{~Hz}$. Furthermore, participants are asked to document their activities in a semi-standardized daily protocol and to answer several paper-based questionnaires about PA and ST after the 7 days of accelerometer use at home.

To assess self-reported PA, the International Physical Activity Questionnaire short form (IPAQ-SF), German version is used. The IPAQ consists of seven items, addressing intensity and duration of PA in daily life over the last 7 days by self-report [35]. Additionally, the German Physical Activity Questionnaire for 50+ (German PAQ 50+) is conducted to assess type and duration of PA in daily life by self-report [36]. Sedentary behavior is captured by the Measure of Older Adults' Sedentary Time (MOST Questionnaire) in the German version, which consists of seven items [37] (Fig. 2).

At baseline all participants receive general information and recommendations about the positive effects of regular PA and less ST on the improvement of cardiovascular risk factors.

Therefore, all study subjects will receive ageappropriate literature from the Federal Centre for Health Education (BZgA) [38] and a chart from the Fonds Gesundes Österreich (FGÖ) [39]. Recommendations provided for PA are based on WHO guidelines for PA for people of age $\geq 65$ years [14].

\section{Random allocation}

After screening and accelerometry, participants will be randomized 1:1 in the intervention and the control group. The randomization will be conducted using an automated function in the documentation software.

\section{Study intervention}

We developed a low-threshold intervention characterized by easy access to the intervention and a low effort for the target group. The intervention comprises two individualized feedback letters, automatically generated in $\mathrm{R}$ software (version 3.3.2, Lucent Technologies, Murray Hill, NJ, USA) from the study data base using the variables number of steps per day, time in minutes of MVPA, and sedentary time in min per day.

Participants in the intervention group will receive a feedback letter by mail shortly after wearing the accelerometer after the baseline examination and after the 3-month follow-up. The feedback letters will contain 


\begin{tabular}{|c|c|c|c|c|c|}
\hline & \multicolumn{5}{|c|}{ STUDY PERIOD } \\
\hline & \multirow{2}{*}{$\begin{array}{c}\text { Enrolment } \\
\text { November } \\
2016 \text { to } \\
\text { December } \\
2017\end{array}$} & \multirow{2}{*}{$\begin{array}{c}\text { Allocation } \\
0 \mathrm{~m}\end{array}$} & \multicolumn{3}{|c|}{$\begin{array}{l}\text { Post-allocation- Intervention } \\
\text { delivery and research follow up }\end{array}$} \\
\hline TIMEPOINT & & & $3 m$ & $6 m$ & $12 m$ \\
\hline \multirow{3}{*}{$\begin{array}{r}\text { ENROLMENT: } \\
\text { Telephone } \\
\text { eligibility screen } \\
\text { Informed consent }\end{array}$} & & & & & \\
\hline & $\mathrm{X}$ & & & & \\
\hline & & $\mathrm{X}$ & & & \\
\hline Allocation & & $X$ & & & \\
\hline \\
\hline \multicolumn{6}{|l|}{$\begin{array}{r}\text { Intervention Group } \\
\text { (Feedback-letter) }\end{array}$} \\
\hline \multicolumn{6}{|l|}{ Control Group } \\
\hline \multirow{3}{*}{$\begin{array}{r}\text { ASSESSMENTS: } \\
\text { Basic socio- } \\
\text { demographic data } \\
\text { General health } \\
\text { status }\end{array}$} & & & & & \\
\hline & & $\mathrm{X}$ & & & \\
\hline & & $\mathrm{X}$ & & $\mathrm{X}$ & $\mathrm{X}$ \\
\hline \multirow{2}{*}{$\begin{array}{r}\text { Blood pressure } \\
\text { Somatometry (BMI, } \\
\text { body weight, waist } \\
\text { and hip } \\
\text { circumference) }\end{array}$} & & $\mathrm{X}$ & $\mathrm{X}$ & $\mathrm{X}$ & $\mathrm{X}$ \\
\hline & & $\mathrm{x}$ & $\mathrm{X}$ & $\mathrm{X}$ & $\mathrm{X}$ \\
\hline Short Form 8 & & $X$ & & $X$ & $\mathrm{X}$ \\
\hline \multirow{2}{*}{$\begin{array}{r}\text { SSA Scale } \\
\text { Mediterranean Diet } \\
\text { questionnaire }\end{array}$} & & $\mathrm{X}$ & $\mathrm{X}$ & $\mathrm{X}$ & $\mathrm{X}$ \\
\hline & & $\mathrm{X}$ & & $\mathrm{X}$ & $\mathrm{X}$ \\
\hline AUDIT & & $\mathrm{X}$ & & $\mathrm{X}$ & $\mathrm{X}$ \\
\hline \multirow{2}{*}{ IPAQ-SF } & & $\mathrm{X}$ & $\mathrm{X}$ & $\mathrm{X}$ & $\mathrm{X}$ \\
\hline & & $\mathrm{X}$ & $\mathrm{X}$ & $\mathrm{X}$ & $\mathrm{X}$ \\
\hline \multirow{2}{*}{$\begin{array}{r}\text { MOST } \\
\text { Accelerometer } \\
\text { device (7 days) }\end{array}$} & & $\mathrm{X}$ & $\mathrm{X}$ & $\mathrm{X}$ & $\mathrm{X}$ \\
\hline & & $X$ & $\mathrm{X}$ & $\mathrm{X}$ & $\mathrm{X}$ \\
\hline
\end{tabular}

Fig. 2 Schedule of enrollment, interventions, and assessments

personalized feedback based on accelerometry as well as ST behavior. PA and ST data will be depicted in three comprehensive graphs:

One graph will represent the number of steps per day that participants took over the 7 days wearing the accelerometer device (Fig. 3).

Therein, 10,000 steps per day will be marked as a red line. Ten thousand steps, has been chosen because this number is used in several prevention programs in Germany and is well known in the population $[40,41]$.
The second graph will constitute intensity of PA divided into colored bar charts per day from red for sedentary activities to dark green for vigorous activities (Fig. 4).

The third graph will show time doing moderate and vigorous activities per week, sitting time per day in percent as a weekly average, count of sit-to-stand transactions as a weekly average, and counts per day as a weekly average measure. To communicate these individual PA and ST measurements effectively to the participants, we will use a multidimensional representation 


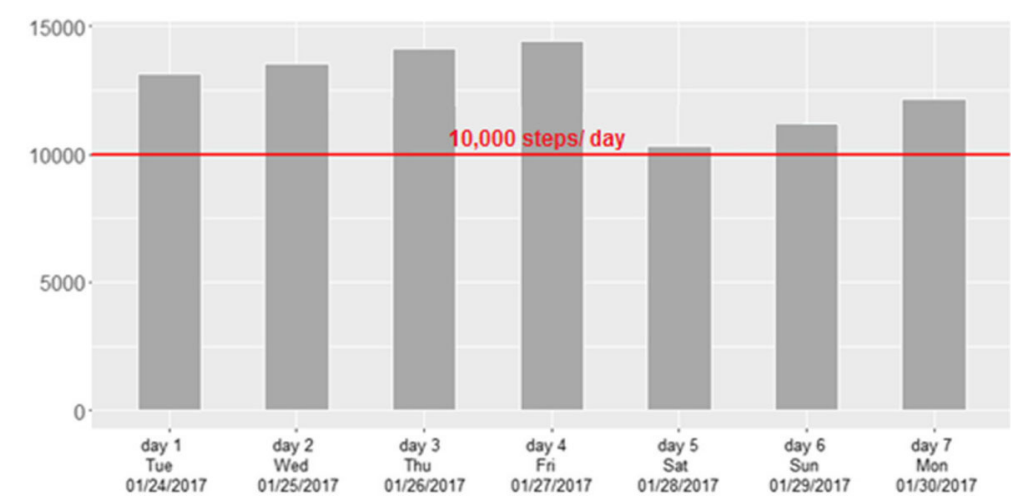

Fig. 3 Graph of steps taken per day

using color bar charts (green represents a high PA level, red represents a low PA level) added with specific recommendations (Fig. 5).

Additionally, participants receive leaflets with ageappropriate recommendations for PA and ST at baseline. The leaflets are from the Federal Centre for Health Education.

\section{Follow-up}

The follow-up examinations after 3, 6, and 12 months will be conducted in the DZHK examination center. Following examination, PA and ST will be objectively measured by the personal accelerometer. After a period of 7 days, participants bring back the accelerometer devices. In addition, study participants receive paper-based questionnaires as shown in the flow chart in Fig. 1.

\section{Power calculation}

The sample size estimate was based on the amount of PA (mean time in min per day), measured in a comparable group of elderly people. In the study in question (Troiano et al.) the amount of PA was on average 15 min of combined PA (moderate and vigorous PA) per day [21]. We took these data as the starting point for the estimation of the required sample size. We assume that participants in the control group remain at approximately the same level while people in the intervention group increase their amount of PA by $20 \%$ [42], which corresponds to an increase from 15 to 18 min of PA per day after 6 months. To demonstrate this effect, a total number of 151 participants is needed (standard deviation $6.0 \mathrm{~min}$, alpha $=0.05$, power $=0.80$ ). Assuming a loss to follow-up of about $30 \%$, we will recruit 216 participants.

\section{Documentation, data storage, data security, and data protection}

Participants' sociodemographics (age, sex, education), lifestyle parameters (nutrition habits, smoking, and alcohol-drinking behavior) from the paper-based questionnaires (self-report) are documented using the Cardiff TeleForm system ${ }^{\circ}$ (Electric Paper, Lüneburg, Germany). The questionnaires and the daily diary protocol contain 1-D barcodes to ensure anonymization. Somatometry data and blood pressure measurements will be documented in eCRFs in an IT-supported documentation system including automatic plausibility and completeness checks [43]. The documentation system is based on the

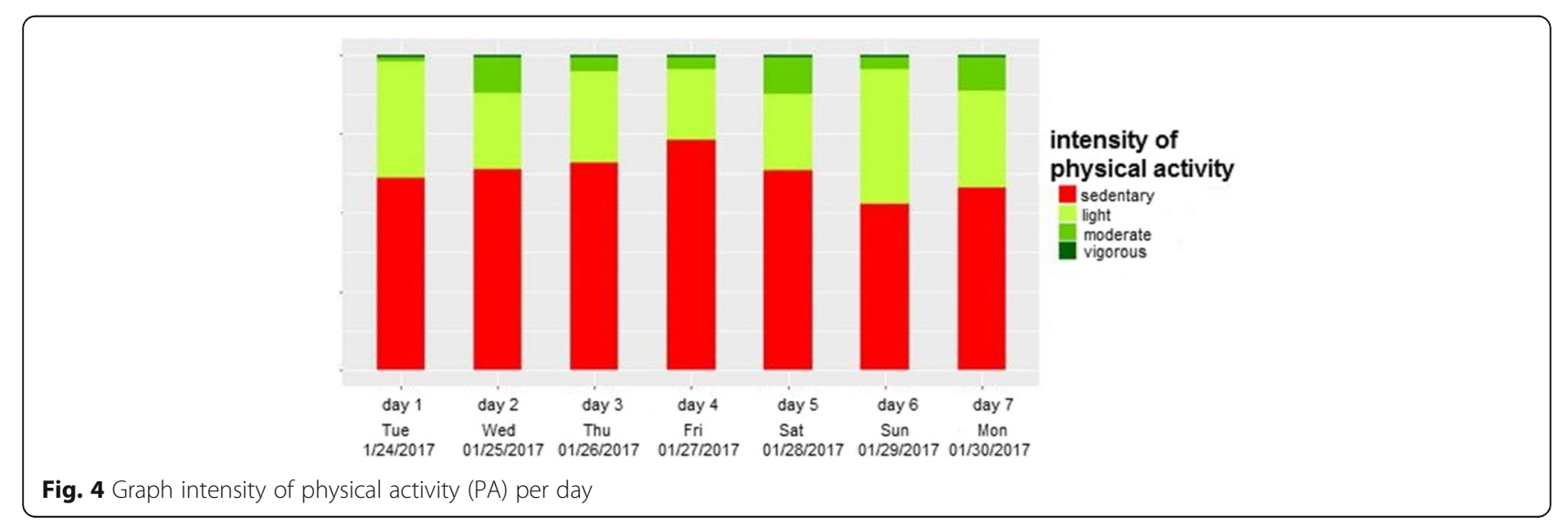


weekly moderate PA on average at least $150 \mathrm{~min}$

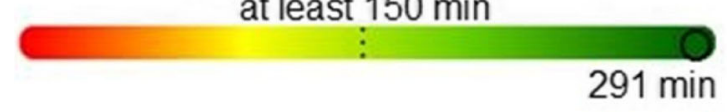

weekly vigorous PA on average at least $75 \mathrm{~min}$

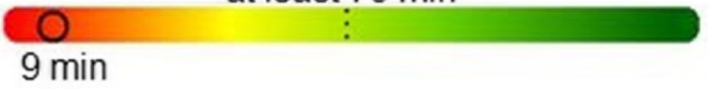

sedentary time per day on average

$\max 60 \%$

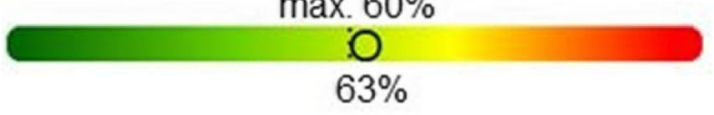

sedentary breaks per day on average at least 20 breaks

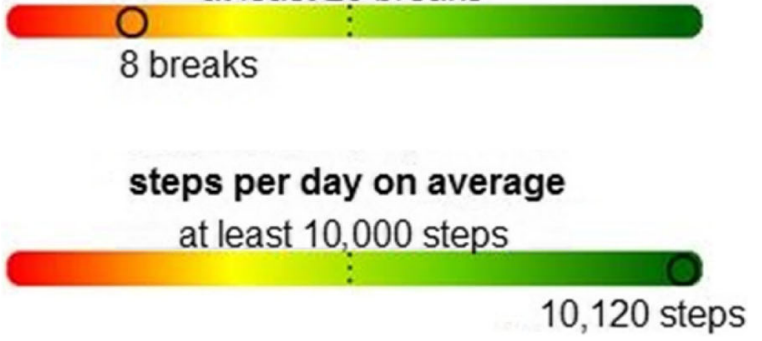

Fig. 5 Graph of specific physical activity (PA) and sitting time (ST)

concept of offline clients, each staff member in the examination center has individual login data [43].

After merging the data assessed in the examination center with the PA data from the accelerometer and the data from the paper-based questionnaires, all data will be stored in a central project data base. The data storage is managed according to current standards for data security and data privacy, documented in the institutional data protection concept of the Institute for Community Medicine [44]. Only the staff members in the examination center have access to personal data during baseline and follow-up examinations.

In addition, all participants receive a study ID card including an 1-D barcode at the baseline examination to support the correct assignment of the participants to their data over time.

\section{Data analysis}

ActiLife software (version 6.13 .2 or later, ActiGraph, Pensacola, FL, USA) will be used for data download and data processing. A valid measurement day is defined as a record of at least $10 \mathrm{~h}$ total wearing time and a record time of at least 4 days is required for data analysis. To categorize PA intensity, we used specific cut points based on Freedson [45]. PA is divided into sedentary (0-99 steps), light (100-1951 steps), moderate (1952-5724 steps), and vigorous (5725-9498 steps) PA. Step counts are based on 60-s epochs.

Statistical analysis will be conducted using pseudonymized data. First, the assessed project data will be descriptively analyzed. We will verify the conditions in both groups. In case of significant differences between the intervention and control group, analyses will be adapted accordingly and adjusted for relevant variables. Subsequently, the primary and secondary outcomes will be analyzed between the groups using $t$ tests or corresponding non-parametric tests in case of ordinal variables or nonnormality of the values. The tests will be conducted based on the intention-to-treat principle. The use of the intention-to-treat procedure ensures that each subject is included in the analysis. The analysis are carried out with the statistical software IBM SPSS Statistics (version 23.0.0.2 or later, IBM Corp., Armonk, NY, USA).

\section{Discussion}

Previous studies have shown positive effects of interventions on PA and ST in older people using accelerometer devices. The interventions included using various different approaches, e.g., face-to-face consultations $[24,26]$ or regular telephone calls [46]. It seems to be difficult to transfer such complex procedures into routine practice due to the higher financial load and personnel expenses incurred.

On the basis of these considerations, our study was initiated to increase PA and reduce ST using a low-threshold intervention by providing automatically generated, individualized feedback letters containing health recommendations and personalized information about each participant's PA and ST behavior. Over the course of the trial study participants twice receive individualized feedback based on accelerometer data. This method seems to be practicable to efficiently reach a high number of persons.

To promote PA in an adequate and lasting way, evidence on possible influencing factors is necessary. Therefore, the potential effect of self-efficacy on PA will be analyzed. Self-efficacy could be a relevant influence factor for changes in PA.

The results of the study will improve our understanding of PA and ST in older people and will provide evidence on how to increase the amount of PA and how to reduce ST behavior. If the intervention is effective, the study has the chance to reduce crucial risk factors for CVD and to improve quality of life, especially in the elderly.

\section{Trial status}

The study started recruitment in November 2016, and is estimated to continue until the end of 2017. The final report will be prepared in 2018. 


\section{Additional file}

Additional file 1: SPIRIT 2013 Checklist. (DOC $120 \mathrm{~kb}$ )

\section{Abbreviations}

BMBF: Federal Ministry of Education and Research; BZgA: Federal Centre for Health Education; CHD: Coronary heart disease; CVD: Cardiovascular diseases: DZHK: German Centre for Cardiovascular Research; eCRF: Electronic care report form; FGÖ: Fonds Gesundes Österreich; German PAQ: German Physical Activity Questionnaire; IPAQ: International Physical Activity Questionnaire; MOST: Measure of Older Adult's Sedentary Time; MVPA: Moderate-tovigorous physical activity; NCD: Non-communicable disease; NHANES: National Health and Nutrition Survey; PA: Physical activity; SF8: Short Form 8; SOP: Standard operating procedures; ST: Sitting time; WHO: World Health Organization

\section{Acknowledgements}

Not applicable

\section{Funding}

The study is funded by the Federal Ministry of Education and Research (BMBF) as a site project of the DZHK. The funders have had no influence on the conceptualization and conduct of the study and will not have any role in the data analysis and publication of the results.

\section{Availability of data and materials}

The final trial dataset will be accessible to the study investigators. Data can be used by other researchers on demand.

\section{Authors' contributions}

The study was conceptualized by FK, FW, TS, MD, KL, SU, WH, and NvdB. $\mathrm{NvdB}$ and $\mathrm{WH}$ are responsible for its overall conduct and are the principal investigators at their respective institutions. TS developed the statistical analyses for the study. FK is the study manager. PP conceptualized and programmed the documentation system. FK drafted the manuscript. All authors have reviewed, provided input to the manuscript, and approved the final manuscript

\section{Ethics approval and consent to participate}

The study was approved by the Ethics Committee of the University Medicine Greifswald (ethic approval BB071/16).

All participants have to provide their written informed consent before they can participate in the study. The original declaration remains in the examination center, participants will receive a copy. Study participants can withdraw their consent any time without negative consequences for them.

\section{Consent for publication}

Not applicable

\section{Competing interests}

The authors declare that they have no competing interests.

\section{Publisher's Note}

Springer Nature remains neutral with regard to jurisdictional claims in published maps and institutional affiliations.

\section{Author details}

${ }^{1}$ Institute for Community Medicine, Section Epidemiology of Health Care and Community Health, University Medicine Greifswald, Greifswald, Germany. 2DZHK (German Centre for Cardiovascular Research), partner site Greifswald, Greifswald, Germany. ${ }^{3}$ Institute of Social Medicine and Prevention, University Medicine Greifswald, Greifswald, Germany. ${ }^{4}$ Department of Internal Medicine B, University Medicine Greifswald, Greifswald, Germany.
Received: 10 March 2017 Accepted: 18 December 2017

Published online: 22 January 2018

\section{References}

1. Marengoni A, Winblad B, Karp A, Fratiglioni L. Prevalence of chronic diseases and multimorbidity among the elderly population in Sweden. Am J Public Health. 2008:98:1198-200.

2. Salive ME. Multimorbidity in older adults. Epidemiol Rev. 2013;35:75-83.

3. Barnett K, Mercer SW, Norbury M, Watt G, Wyke S, Guthrie B. Epidemiology of multimorbidity and implications for health care, research, and medical education: a cross-sectional study. Lancet. 2012;380:37-43.

4. Gößwald A, Schienkiewitz A, Nowossadeck E, Busch MA. Prevalence of myocardial infarction and coronary heart disease in adults aged $40-79$ years in Germany. Bundesgesundheitsblatt Gesundheitsforschung Gesundheitsschutz. 2013;56:650-5.

5. Lozano R, Naghavi M, Foreman K, Lim S, Shibuya K, Aboyans V, et al. Global and regional mortality from 235 causes of death for 20 age groups in 1990 and 2010: a systematic analysis for the Global Burden of Disease Study 2010. Lancet. 2012;380:2095-128.

6. Statistisches Bundesamt. Gestorbene nach ausgewählten Todesursachen. Anzahl der Gestorbenen 2014. 2016. https://www.destatis.de/DE/ ZahlenFakten/GesellschaftStaat/Gesundheit/Todesursachen/Tabellen/ EckdatenTU.html. Accessed 10 Nov 2016.

7. Miles L. Physical activity and health. Nutr Bull. 2007;32:314-63.

8. Yusuf S, Reddy S, Ounpuu S, Anand S. Global burden of cardiovascular diseases: part l: general considerations, the epidemiologic transition, risk factors, and impact of urbanization. Circulation. 2001;104:2746-53.

9. Lohne-Seiler $\mathrm{H}$, Hansen $\mathrm{BH}$, Kolle $\mathrm{E}$, Anderssen SA. Accelerometer-determined physical activity and self-reported health in a population of older adults (65-85 years): a cross-sectional study. BMC Public Health. 2014;14:1-10.

10. Krug S, Jordan S, Mensink GBM, Müters S, Finger J, Lampert T. Physical activity. Bundesgesundheitsblatt Gesundheitsforschung Gesundheitsschutz. 2013:56:765-71.

11. Allender S, Foster C, Boxer A. Occupational and nonoccupational physical activity and the social determinants of physical activity: results from the Health Survey for England. J Phys Act Health. 2008;5:104-16.

12. Löllgen $H$, Löllgen D. Risk reduction in cardiovascular diseases by physical activity. Internist. 2012;53:20-9.

13. World Health Organization. Global Recommendations on Physical Activity for Health: 18-64 years old. 2011. http://www.who.int/dietphysicalactivity/physicalactivity-recommendations-18-64years.pdf?ua=1. Accessed 27 Oct 2016.

14. World Health Organization. Global Recommendations on Physical Activity for Health: 65 years and above. 2011. http://www.who.int/ dietphysicalactivity/physical-activity-recommendations-65years.pdf?ua=1. Accessed 27 Oct 2016

15. Hootman JM. 2008 Physical activity guidelines for Americans: an opportunity for athletic trainers. J Athl Train. 2009:44:5-6.

16. Colley RC, Garriguet D, Janssen I, Craig CL, Clarke J, Tremblay MS. Physical activity of Canadian adults: accelerometer results from the 2007 to 2009 Canadian Health Measures Survey. Health Rep. 2011:22:7-14.

17. Hawkins MS, Storti KL, Richardson CR, King WC, Strath SJ, Holleman RG, et al. Objectively measured physical activity of USA adults by sex, age, and racial/ ethnic groups: a cross-sectional study. Int J Behav Nutr Phys Act. 2009;6:31.

18. Sun F, Norman IJ, While AE. Physical activity in older people: a systematic review. BMC Public Health. 2013;13:1-17.

19. Matthews CE, Chen KY, Freedson PS, Buchowski MS, Beech BM, Pate RR, et al. Amount of time spent in sedentary behaviors in the United States, 20032004. Am J Epidemiol. 2008;167:875-81.

20. Godfrey A, Lord S, Galna B, Mathers JC, Burn DJ, Rochester L. The association between retirement and age on physical activity in older adults. Age Ageing. 2014;43:386-93.

21. Troiano RP, Berrigan D, Dodd KW, Masse LC, Tilert T, McDowell M. Physical activity in the United States measured by accelerometer. Med Sci Sports Exerc. 2008;40:181-8.

22. Harvey JA, Chastin SF, Skelton DA. How sedentary are older people? A systematic review of the amount of sedentary behavior. J Aging Phys Act. 2015:23:471-87.

23. Gaede-Illig C, Zachariae S, Menzel C, Alfermann D. Körperliche Aktivität erfassen - ein Vergleich vom IPAQ-SF und dem SenseWear Pro Armband. Dtsch Z Sportmed. 2014;65:154-9. 
24. Gardiner PA, Eakin EG, Healy GN, Owen N. Feasibility of reducing older adults' sedentary time. Am J Prev Med. 2011;41:174-7.

25. Rosenberg DE, Gell NM, Jones SM, Renz A, Kerr J, Gardiner PA, et al. The feasibility of reducing sitting time in overweight and obese older adults. Health Educ Behav. 2015;42:669-76.

26. Harris $T$, Kerry $S M$, Victor CR, Ekelund U, Woodcock A, lliffe $S$, et al. A primary care nurse-delivered walking intervention in older adults: PACE (Pedometer Accelerometer Consultation Evaluation)-Lift cluster randomised controlled trial. PLoS Med. 2015;12:e1001783.

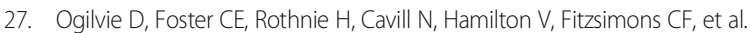
Interventions to promote walking: systematic review. BMJ. 2007;334(7605):1204.

28. Kang M, Marshall SJ, Barreira TV, Lee JO. Effect of pedometer-based physical activity interventions: a meta-analysis. Res Q Exerc Sport. 2009;80:648-55.

29. Bravata DM, Smith-Spangler C, Sundaram V, Gienger AL, Lin N, Lewis R, et al. Using pedometers to increase physical activity and improve health: a systematic review. JAMA. 2007;298:2296-304.

30. Chan AW, Tetzlaff JM, Altman DG, Laupacis A, Gotzsche PC, Krleza-Jeric K, et al. SPIRIT 2013 statement: defining standard protocol items for clinical trials. Ann Intern Med. 2013;158:200-7.

31. Ellert U, Lampert T, Ravens-Sieberer U. Measuring health-related quality of life with the SF-8. Bundesgesundheitsblatt Gesundheitsforschung Gesundheitsschutz. 2005:48:1330-7.

32. Fuchs R, Schwarzer R. Selbstwirksamkeit zur sportlichen Aktivität: Reliabilität und Validität eines neuen Meßinstruments. Z Differ Diagn Psychol. 1994;15:141-54.

33. Schroder H, Fito M, Estruch R, Martinez-Gonzalez MA, Corella D, SalasSalvado J, et al. A short screener is valid for assessing Mediterranean diet adherence among older Spanish men and women. J Nutr. 2011;141:1140-5.

34. Schweizerische Koordinations- und Fachstelle Sucht. Alter und Sucht. Alcohol Use Disorders Identification Test (AUDIT). https://www.alterundsucht.ch/de/ aerztinnen/alkohol/screening/audit. Accessed 5 Nov 2016.

35. The International Physical Activity Questionnaire group. International Physical Activity Questionnaire. Short Last 7 Days Self-Administered Format-German Version. 2016. https://docs.google.com/viewer?a=v\&pid=sites\&srcid= ZGVmYXVsdGRvbWFpbnxOaGVpcGFxfGd4OjNiMGRjYjU3NzE5Nzc0ZGY Accessed 10 Oct 2016.

36. Leibnitz-Zentrum für Psychologische Information und Dokumentation (ZPID). German-PAQ-50+: Fragebogen zur Erfassung der körperlichen Aktivität. 2015. https://www.zpid.de/pub/tests/PT_9006067_German-PAQ-50 +_Fragebogen.pdf. Accessed 9 Oct 2016.

37. Gardiner PA, Clark BK, Healy GN, Eakin EG, Winkler EA, Owen N. Measuring older adults' sedentary time: reliability, validity, and responsiveness. Med Sci Sports Exerc. 2011;43:2127-33.

38. Bundeszentrale für gesundheitliche Aufklärung. Gesundheit älterer Menschen. Alltag in Bewegung. 2014. http://www.aelter-werden-in-balance. de/fileadmin/user_upload/dokumente/Alltag_in_Bewegung_24_final.pdf. Accessed 11 Nov 2016.

39. Fonds Gesundes Österreich. Bewegungsempfehlungen für Erwachsene. 2012. http://www.fgoe.org/presse-publikationen/downloads/fotos-grafiken/ infografiken/Grafik-der-Bewegungsempfehlungen-fuer-Erwachsene/2012-1005.4766199807/view. Accessed 11 Nov 2016.

40. Bayrisches Landesamt für Gesundheit und Lebensmittelsicherheit. 10.000 Schritte. Eine Aktion der SBK für alle, die mehr Bewegung in ihren Alltag bringen wollen. 2007. http://www.zpg-bayern.de/id-10000-schritte.html. Accessed 6 Dec 2017.

41. Sportärztebund Nordrhein e.V. Kampagne "10,000 Schritte für thre Gesundheit." 2016. https://www.sportaerztebund.de/36-kampagne-10-000schritte-fuer-ihre-gesundheit. Accessed 6 Dec 2017.

42. Tudor-Locke C, Craig CL, Aoyagi Y, Bell RC, Croteau KA, De Bourdeaudhuij I, et al. How many steps/day are enough? For older adults and special populations. Int J Behav Nutr Phys Act. 2011;8:80.

43. Meyer J, Fredrich D, Piegsa J, Habes M, van den Berg N, Hoffmann W. A mobile and asynchronous electronic data capture system for epidemiologic studies. Comput Methods Programs Biomed. 2013;110:369-79.

44. Bialke M, Penndorf P, Wegner T, Bahls T, Havemann C, Piegsa J, et al. A workflow-driven approach to integrate generic software modules in a Trusted Third Party. J Transl Med. 2015;13:176.

45. Freedson PS, Melanson E, Sirard J. Calibration of the Computer Science and Applications, Inc. accelerometer. Med Sci Sports Exerc. 1998;30:777-81.

46. Lawton BA, Rose SB, Raina Elley C, Dowell AC, Fenton A, Moyes SA. Exercise on prescription for women aged 40-74 recruited through primary care: two year randomised controlled trial. Br J Sports Med. 2009;43:120-3.

\section{Submit your next manuscript to BioMed Central and we will help you at every step:}

- We accept pre-submission inquiries

- Our selector tool helps you to find the most relevant journal

- We provide round the clock customer support

- Convenient online submission

- Thorough peer review

- Inclusion in PubMed and all major indexing services

- Maximum visibility for your research

Submit your manuscript at www.biomedcentral.com/submit 